\title{
Anterior decompression for cervical spondylosis associated with an early form of cervical ossification of the posterior longitudinal ligament
}

\author{
Junichi Mizuno, M.D., ANd Hiroshi Nakagawa, M.D. \\ Department of Neurological Surgery, Aichi Medical University, Aichi, Japan
}

\begin{abstract}
Object. The goal of this study was to determine the appropriate surgical strategy for cervical spondylosis associated with an early form of ossification of the posterior longitudinal ligament (EOPLL) of the cervical spine.

Methods. Patients with EOPLL-associated cervical spondyosis were selected for treatment. Medical records and radiographs were retrospectively reviewed. Specimens taken at the time of operation were histologically examined. There were 24 men and six women ranging in age from 39 to 74 years (mean 57.6 years). Symptoms consisted of myelopathy in 28 cases and radiculopathy in two cases. Anterior decompressive surgery was performed. The EOPLL, hypertrophy of the posterior longitudinal ligament (HPLL), and the disc-PLL complex were directly resected. The mean preoperative Japan Orthopaedic Association score was 12.6, and the mean postoperative score was 14.4 Histologically, EOPLL was consistent with foci of compact lamellar bone in the degenerative thickening of the PLL.

Conclusions. Appropriate corpectomy should follow direct removal of EOPLL associated with HPLL compressing the spinal cord to achieve good outcomes.
\end{abstract}

KEY WordS - posterior longitudinal ligament • ossification • hypertrophy • cervical spondylosis • corpectomy

Cervical spondylosis associated with EOPLL or HPLL may cause symptomatic myelopathy, radiculopathy, or radiculomyelopathy. Although OPLL, radiographically classified as segmental, continuous, mixed, and other type by Hirabayashi and colleagues ${ }^{9}$ is essentially treated by an anterior approach with direct removal of the OPLL, $2,4,6,8$, $15,17,19,20$ surgical treatment of EOPLL or HPLL in which cervical spondylosis forms the symptomatic disc-PLL complex has not been well discussed. Only a few investigators have paid attention to the disc-PLL complex as an important factor in the cervical cord compression. ${ }^{3,5,16 \text {, }}$ 20,22,23,35 Unlike cases with classic OPLL, prominent bone formation is not found in EOPLL associated with cervical spondylosis. Cord compression caused by the disc-PLL complex, however, extends to the midvertebral level as well as to the vertebral endplate level. Therefore, anterior discectomy performed through the disc spaces fails to yield a good outcome in patients with the disc-PLL complex. Precise radiological evaluation of the disc-PLL complex leads to correct diagnosis and treatment.

Abbreviations used in this paper: $\mathrm{CT}=$ computerized tomography; EOPLL = early-form ossification of the posterior longitudinal ligament; HPLL = hypertrophy of the PLL; JOA = Japan Orthopaedic Association; $\mathrm{MR}=$ magnetic resonance; $\mathrm{VB}=$ vertebral body; $3 \mathrm{D}=$ three-dimensional.
In this report, we describe the radiological characteristics of EOPLL-associated cervical spondylosis cord compression and discuss the optimum operation to achieve better outcomes.

\section{CLINICAL MATERIAL AND METHODS Patient Population}

Between August 1997 and April 2001, CT and MR imaging were used to diagnose cervical spondylosis and EOPLL associated with HPLL (the disc-PLL complex) in 30 patients. There were 24 men and six women, who ranged in age from 39 to 74 years (mean 57.6 years). Symptoms included myelopathy in 28 and radiculopathy in two cases. After anterior decompressive surgery with direct removal of the disc-PLL complex, interbody cage fixation was performed in all cases. The minimum followup period was 6 months.

\section{Radiological Examination}

Plain radiographs, polytomography scans, and CT studies were obtained in all patients. Polytomography and CT scans were reviewed in relation to cervical spondylosis, EOPLL, and HPLL. In 20 patients MR imaging was performed. Sagittal and transverse $T_{1}$ - and $T_{2}$-weighted sequences were obtained. The MR images were assessed in 
relation to cervical spondylosis, EOPLL, HPLL, and the degree of cord compression.

\section{Operative Procedure}

With the help of an operating microscope, drilling of the osteophyte and EOPLL and en bloc removal of residual OPLL and HPLL were performed by appropriate corpectomy; fixation was achieved by placing titanium interbody cages (porous CCM; A-Spine, Tokyo, Japan). The cylindrical-shaped threaded interbody fusion cages are available in sizes from 6 to $14 \mathrm{~mm}$ in inner diameter. We placed a single cage larger than $10 \mathrm{~mm}$ in the corpectomy defect and twin cages smaller than $8 \mathrm{~mm}$ for the discogenic disease. After reaming of the adjacent VBs following appropriate discectomy, decompression was performed by removing the EOPLL and HPLL (the disc-PLL complex). The interbody fusion cage was filled with bone chips obtained from the vertebrae or with hydroxyapatite granules and then inserted into the space as a graft. Postoperatively, the patients wore a cervical soft collar for 4 weeks. Follow-up examinations were performed 6 months after the operation.

\section{Pathological Examination}

The surgical specimens were analyzed using hematoxylin and eosin staining to confirm the diagnosis and to analyze the associated pathological changes of the ligament.

\section{RESULTS}

\section{Surgery-Related Outcomes}

The anterior approach was used to remove the EOPLL and HPLL, and subsequent reconstruction of the cervical column was performed in all cases. The mean preoperative JOA score (Table 1) was 12.6, and the JOA score increased to 14.4 postoperatively (out of a possible score of 17). There were no cases of neurological deterioration after the operations. No spinal cord or nerve root injuries or infections were demonstrated, although minor cerebrospinal fluid leakage occurred in two cases. A small dural laceration was repaired by placing fascia and Surgicel combined with fibrin glue. Satisfactory removal of the lesions was achieved through the space of an appropriate corpectomy, and no second operation for further decompression was necessary. Of 21 patients in whom with a minimum of 1 year follow-up data were available, fusion was achieved in 95\%. The following criteria were used to indicate successful fusion: 1) the absence of motion on flexion-extension radiographs and 2) the absence of any dark halo around a cage on both anteroposterior and lateral radiographic views.

\section{Radiological Evaluation}

Identification of EOPLL was quite problematic on plain lateral radiographs because of overlapping of posterior osteophytes and focal ossification at the posterior margin of the vertebral body. On CT scans, EOPLL was indicated by a high density mass at the central posterior margin of the VB, whereas HPLL was demonstrated as slightly high to isodensity mass. Sagittal reformations of 3D CT scans were useful for identifying the longitudinal exten-
TABLE 1

Summary of classifications in the JOA system

I) upper extremity motor function

0 impossible to eat $\mathrm{w} /$ either chopsticks or spoon

1 possible to eat w/ spoon but not w/ chopsticks

2 possible to eat $w /$ chopsticks but inadequate

3 possible to eat w/ chopsticks but awkward

4 normal

II) lower extremity motor function

0 impossible to walk

1 need cane or aid on flat ground

2 need cane or aid on stairs

3 possible to walk w/out cane or aid but slow

4 normal

III) sensory function

A) upper extremies

0 apparent sensory loss

1 minimal sensory loss

2 normal

B) lower extremities

0 apparent sensory loss

1 minimal sensory loss

2 normal

C) trunk

0 apparent sensory loss

1 minimal sensory loss

2 normal

IV) bladder function

0 urinary retention/overflow incontinence

1 severe dysuria/sense of retention/retardation

2 slight dysuria/pollakiuria/protraction

3 normal sion of EOPLL originating at the posterior margin of the VB. On $T_{1}$-weighted MR images, EOPLL and the spondylosis mass were revealed as hypointense lesions, whereas HPLL was observed as an isointense lesion. The quality of $\mathrm{T}_{1}$-weighted $\mathrm{MR}$ imaging was superior to that of $\mathrm{T}_{2}$-weighted imaging in the differentiation of the spondylotic lesion, EOPLL, and HPLL contributing the cord compression. Sagittal MR imaging clearly revealed the expansion of cord compression due to the disc-PLL complex at the mid-VB levels beyond the disc spaces. The most severe cord compression was seen at the disc spaces. We have attempted a classification of OPLL of the cervical spine including EOPLL on the basis of these radiological findings (Fig. 1).

\section{Histological Evaluation}

Twelve specimens were evaluated to determine the pathological changes of the disc-PLL complex. Examination of the cervical spondylosis associated with the EOPLL showed herniated intervertebral discs bulging into the deep layer of the PLL with relatively clear demarcation. The PLL contained foci of ossification and associated chondroid tissue in which hyalinoid degeneration was demonstrated. There was prominent assembly of small vessels between the deep and superficial layers of the ligament. The margin between ossification and subsequent HPLL was indistinct. Interestingly, ossification was more frequent in the superficial layer rather than the deep layer of the ligament. 


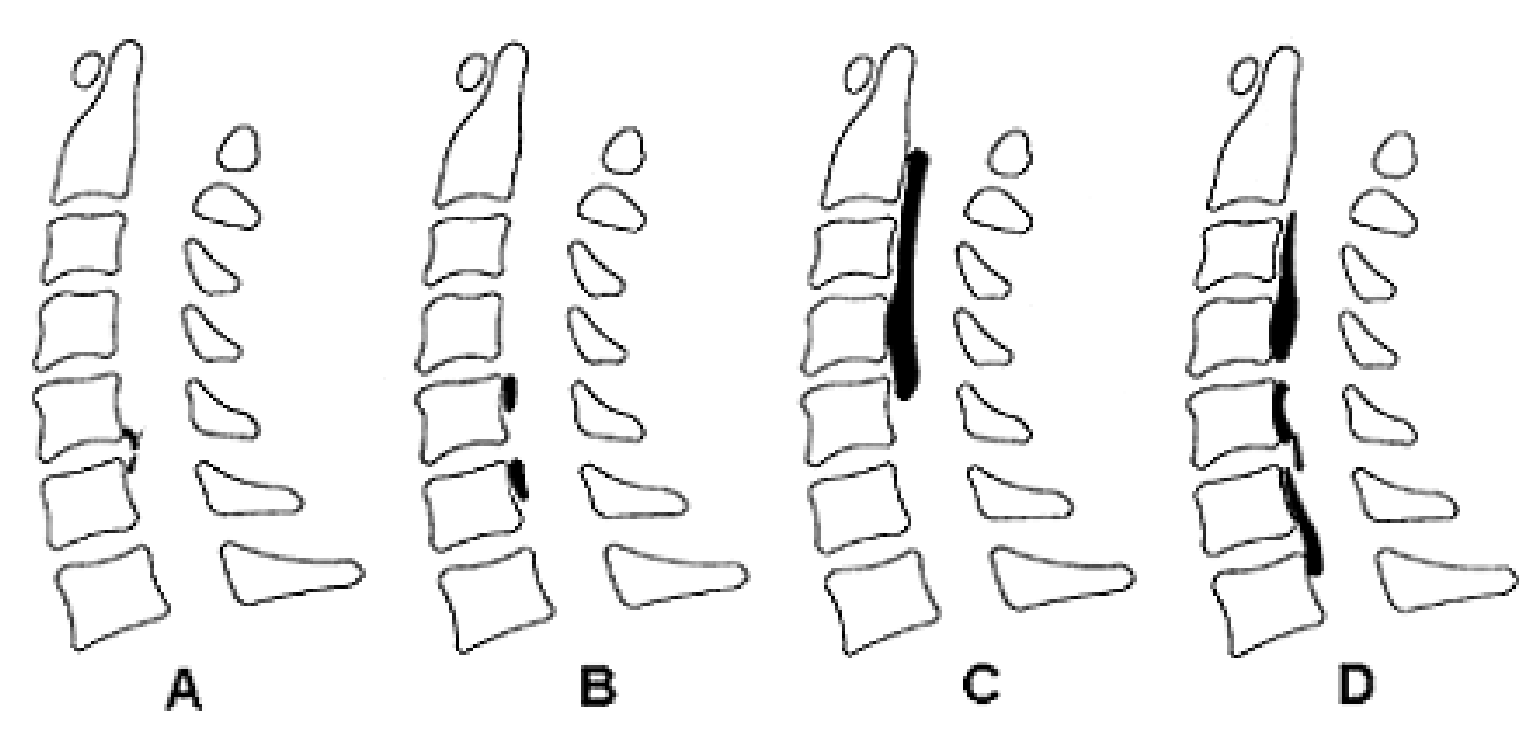

Fig. 1. Schematic drawings of radiological characteristics of classic OPLL and EOPLL. A: Early OPLL (focal ossification at the posterior margin of VB. B: Segmental OPLL (ossification behind each VB). C: Continuous OPLL (sticklike ossification over two VBs). D: Mixed OPLL (combined segmental OPLL and continuous OPLL).

\section{ILLUSTRATIVE CASES}

\section{Case 1}

This 54-year-old man was admitted to our hospital with a 3-month history of progressive gait disturbance. Neurological examination demonstrated weakness of wrist extension and flexion, clumsiness of fingers, numbness below C-7, hyperreflexia of knee and ankle jerks, and spastic gait. His preoperative JOA score was 9. Cervical radiography revealed spondylotic change at C5-6. A CT study demonstrated a bilateral osteophytic spur at C5-6 and small EOPLL at C-6. An MR imaging study disclosed a herniated intervertebral disc, osteophytic spur, OPLL, and associated HPLL causing increased cord compression from C-5 to C-6. Anterior decompression was performed in which the disc-PLL complex was removed and a 12$\mathrm{mm}$-diameter titanium interbody fusion cage was placed (Fig. 2). The 6-month follow-up JOA score was 13. The surgical specimen showed thickened PLL with ossification and hyalinoid degeneration with ruptured disc material (Fig. 3).

\section{Case 2}

This 51-year-old man was admitted to our hospital with an 8-month history of upper-extremity numbness. Neurological examination demonstrated clumsiness of fingers, hypesthesia in the C-7, C-8, and T-1 region, hyperreflexia in the knee and ankle jerks, and spastic gait. His preoperative JOA score was 12. Cervical radiograph revealed loss of lordosis and spondylotic changes at C4-5, C5-6, and C6-7. Both CT and 3D CT scans demonstrated EOPLL at C-5 and C-6 and HPLL at C-5. An MR imaging study disclosed cord compression at the $\mathrm{C} 4-5$ disc space and extensive cord compression at C5-6. Anterior decompression was performed in which the C5-6 disc-PLL complex and C4-5 osteophyte were excised. Placement of two 7-mmdiameter cages at $\mathrm{C} 4-5$ and a single $12-\mathrm{mm}$-diameter cage at C5-6 was then performed (Fig. 4). The patient's 6-month JOA score was 16. Examination of the surgical specimen showed thickened PLL with focal ossification and degenerated ligamentous fibers (Fig. 5).

\section{DISCUSSION}

\section{Pathophysiology of OPLL}

Ossification of the posterior longitudinal ligament in the cervical spine is a well-known clinical entity that causes symptomatic spinal cord compression, not only in Japan but also in North America. ${ }^{17,15,18,19,21,25,26,31}$ Although many investigators have proposed various theories regarding the formation of OPLL, ${ }^{10,12,13,14,18,28-30,32,34}$ the exact induction mechanism of ossification in the PLL remains unclear. We are not even certain of the difference among each of the four types of OPLL (segmental, continuous, mixed, others), ${ }^{9}$ nor how segmental OPLL grows into continuous or mixed OPLL. One of the characteristics of segmental OPLL is its association with herniated intervertebral discs and cervical spondylotic changes. ${ }^{32-34}$ This phenomenon supports the assumption that the local mechanical stress imposed on the PLL by compression of herniated intervertebral discs together with minor neck trauma might cause the PLL to degenerate; enchondral ossification may be subsequently induced at the posterior margin of the VB. Ultimately, this ossification becomes radiographically evident as segmental OPLL behind the VB.

\section{The Disc-Posterior Longitudinal Ligament Complex}

Apart from classic segmental OPLL, symptomatic focal ossification at the posterior margin of the VBs has been documented as OPLL in evolution, ${ }^{5}$ HPLL, ${ }^{20}$ and advanced cervical spondylosis with EOPLL. ${ }^{3}$ The concept of these abnormalities of the disc-PLL complex implies thickened PLL containing foci of ossification together with cervical spondylosis, which cause compression of the spinal cord not only at the endplate level but also in the 


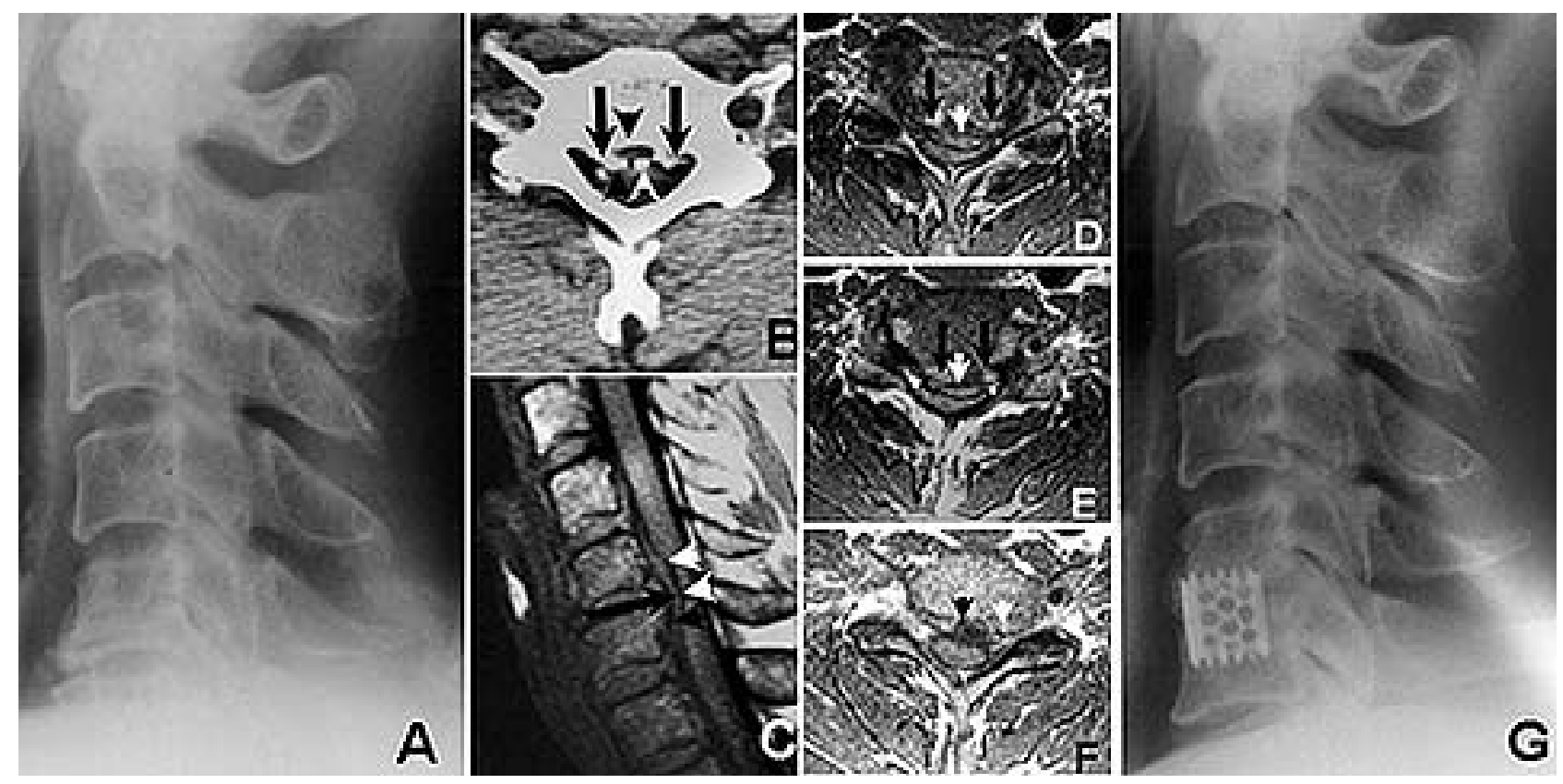

Fig. 2. Case 1. A: Cervical radiograph demonstrating narrowing of the disc space at C5-6 with spondylotic change. B: Axial CT scan demonstrating bilateral posterior osteophyte (arrows) with EOPLL (arrowhead) in the PLL (white arrowhead) at C-6. C: Sagittal $\mathrm{T}_{1}$-weighted MR image revealing extensive cord compression from C-5 to C-6 due to the disc-PLL complex (posterior osteophyte [arrow], EOPLL [arrowhead], and HPLL [white arrowhead]). D-F: Transverse $T_{1}$-weighted MR images obtained at C-5 (D), C5-6 (E), and C-6 (F). G: Postoperative cervical radiograph demonstrating satisfactory removal of the disc-PLL complex and fusion with an interbody cage.

midvertebral level. The most striking difference in the anatomical location between EOPLL and classic segmental OPLL is that EOPLL is formed focally at the posterior margin of the VBs, whereas classic segmental OPLL occurs behind the VBs. To understand and identify EOPLL more thoroughly, we want to emphasize the introduction of EOPLL associated with cervical spondylosis as an initial formation of ossification in the PLL as shown in Fig. 1.

In the patients in our series with cervical spondylosis associated with EOPLL no significant differences were shown from classic OPLL in terms of age, sex, preoperative symptoms, or surgery-related outcomes, ${ }^{15,20,24,26}$ although Epstein ${ }^{3,5}$ has reported that patients with OPLL in evolution were significantly younger than those with classic OPLL and that the male/female ratio was approximately $2: 1$. We assume that EOPLL is an ultimate product of degenerated PLL stimulated by bulging of a herniated intervertebral disc, which is commonly observed in the middle-aged population. Thus, cervical spondylosis associated with EOPLL is observed more frequently in the elderly rather than younger populations.

\section{Radiological Manifestations}

It is essential to evaluate the CT findings of the discPLL complex, particularly those observed on 3D CT studies. Plain lateral cervical radiographs are least useful to identify EOPLL and HPLL in association with cervical spondylosis. The $\mathrm{T}_{2}$-weighted MR imaging studies poorly differentiated EOPLL from cervical spondylosis because of similarities in signal intensity. Magnetic resonance imaging in which $\mathrm{T}_{1}$-weighted sequences are obtained is useful to differentiate each component of the disc-PLL complex. Hypertrophy of the PLL contributing to the

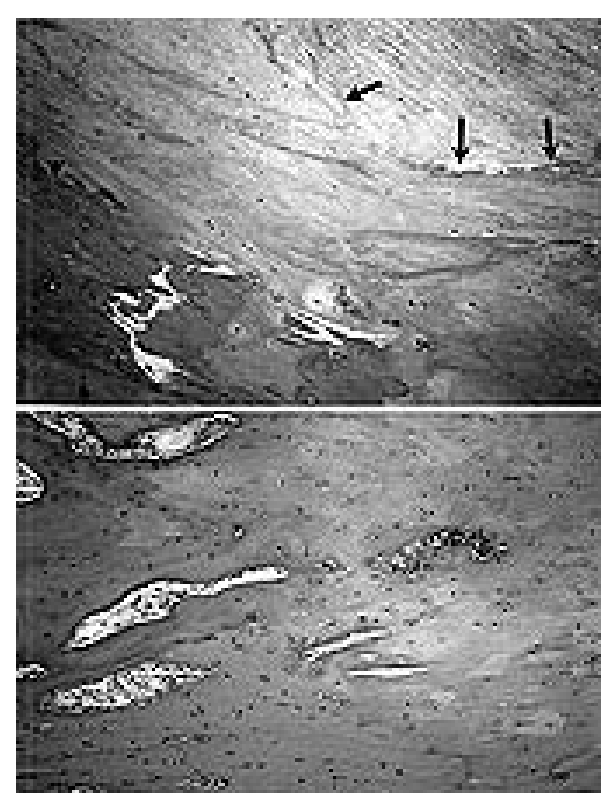

Fig. 3. Case 1. Photomicrographs. Upper: Herniated intervertebral disc into the deep layer of the PLL with relatively clear margin (arrows). There is hyalinoid degeneration in the deep layer and focal ossification in the superficial layer (arrowheads). H \& E, original magnification $\times 4$. Lower: Proliferation of small vessels is seen between the deep and superficial layers of the PLL. H \& E, original magnification $\times 20$. 


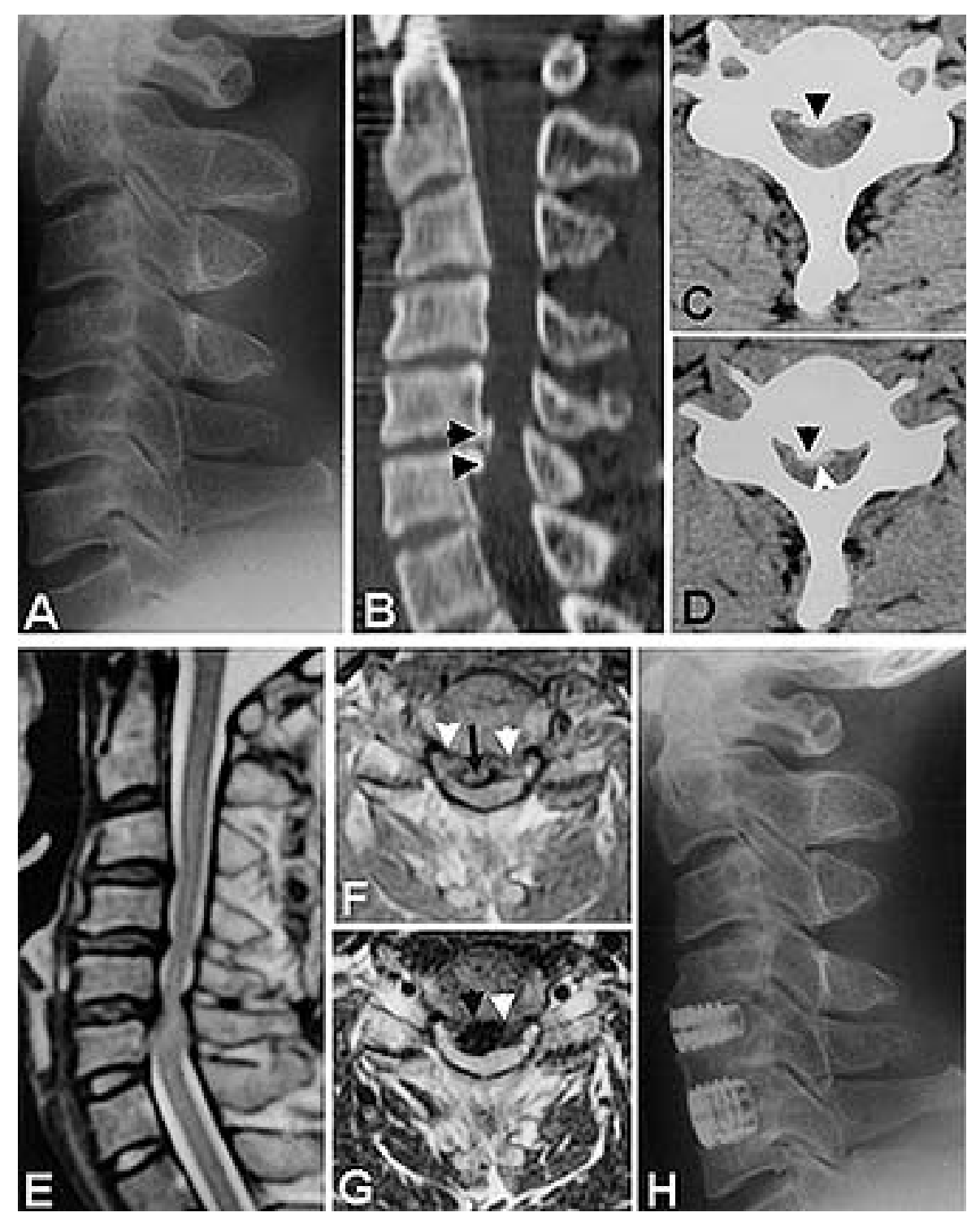

Fig. 4. A: Cervical radiograph revealing kyphotic deformity with moderate spondylotic change at C4-5 and C5-6. B-D: Sagittal and axial 3D CT and CT studies demonstrating: EOPLL (arrowheads) at the posterior margin of C-5 and C-6 VBs, posterior osteophytes (arrows) and HPLL (white arrowheads) at C-5. E: Sagittal T,-weighted MR image revealing moderate cord compression due to a spondylotic lesion at $\mathrm{C} 4-5$ and extensive cord compression with intramedullary high intensity due to spondylosis and EOPLL with associated HPLL at C5-6. F and G: Transverse $\mathrm{T}_{1}-$ weighted MR images demonstrating deformity of spinal cord due to the disc-PLL complex that consists of herniated intervertebral disc (arrow), EOPLL (arrowhead), and HPLL (white arrowhead). H: Postoperative cervical radiograph after removal of the osteophyte at C4-5 and the C5-6 disc-PLL complex and interbody cage fixation.

expansion of the cord compression is revealed as a slightly high-intensity or isointensity signal on $\mathrm{T}_{1}$-weighted MR imaging or a slightly higher density mass than that of OPLL on CT studies. Meticulous evaluation of neuroimaging-documented HPLL associated with EOPLL and cervical spondylosis with extended cord compression is essential to differentiate the reactive focal thickening of the PLL only at the disc space, which is commonly seen in cases with discogenic disease.

\section{Choice of Surgical Procedure}

In cases of cervical spondylosis anterior decompressive surgery and fusion is mandatory for resection of EOPLL. Although EOPLL appears as a focal mass of the posterior margin of the VBs on neuroimaging, associated pathological thickening of the PLL is common. ${ }^{3,20}$ Therefore, microsurgical direct excision of the disc-PLL complex by central vertebrectomy should be performed and followed by interbody cage fixation. The PLL may be incised after discectomy if it is incomplete to obtain satisfactory decompression. Although we performed anterior procedures in all cases because they were one- to two-segment EOPLL, we may perform laminectomy or laminoplasty in multisegmental cases in which more than three levels are 


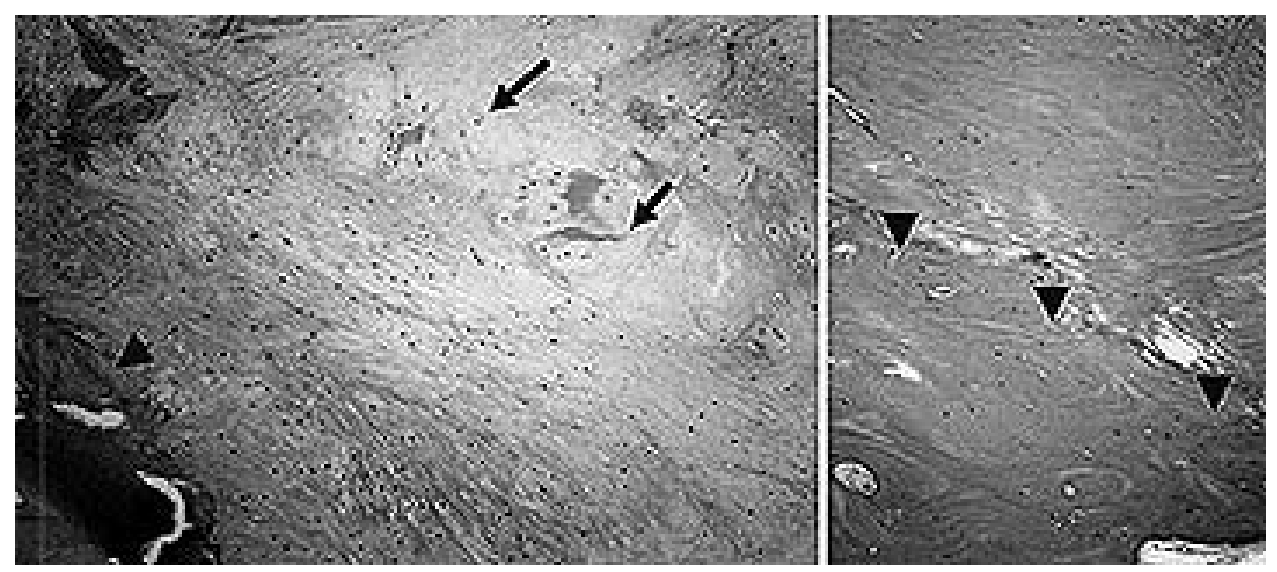

Fig. 5. Case 2. Photomicrographs. Left: Herniated intervertebral disc into the deep layer of the PLL (arrows). Degenerated PLL with calcification was found in the deep layer and focal EOPLL in the deep layer (arrowhead). H \& $\mathrm{E}$, original magnification $\times 4$. Right: Ossification is observed in the superficial layer (arrowheads). $\mathrm{H} \& \mathrm{E}$, original magnification $\times 12$.

involved. Progression of OPLL after laminoplasty or laminectomy, however, has been reported by the several authors; $9,11,27,36$ therefore, we suggest that EOPLL in cases of cervical spondylosis involving fewer than two levels should be treated by anterior procedures to avoid symptomatic progression or recurrence of OPLL.

\section{Microsurgical Removal of EOPLL}

We encountered two cases of minor cerebrospinal fluid leakage during removal of the disc-PLL complex from the underlying dura mater. Long-standing dural compression by EOPLL and HPLL may show dense adhesion with the dura mater. Careful attention should be paid to microsurgical separation of EOPLL and HPLL from the dura. The area of ossification was focal but frequently associated with HPLL rostally and caudally. Therefore, appropriate corpectomy is necessary, and the residual compression behind the midvertebral portion should be identified by inserting angulated curettes or microdissecters after removal of the disc-PLL complex at the corpectomy site. Unlike classic OPLL, EOPLL did not extend into the farlateral portion of the spinal canal, and C-5 paresis was not encountered in our series.

\section{CONCLUSIONS}

Cervical spondylosis associated with EOPLL may be categorized as an early form of classic segmental OPLL. This lesion frequently coexists with subsequent HPLL. The disc-PLL complex makes one unit that compresses the spinal cord more extensively and severely than simple cervical spondylosis in which there is focal reactive degeneration of the PLL. An appropriate corpectomy is essential for satisfactory decompression in this clinical entity.

\section{References}

1. Abe H, Tsuru M, Ito T, et al: Anterior decompression for ossification of the posterior longitudinal ligament of the cervical spine. J Neurosurg 55:108-116, 1981
2. Bakay L, Cares HL, Smith RJ: Ossification of the region of the posterior longitudinal ligament as a cause of cervical myelopathy. J Neurol Neurosurg Psychiatry 33:263-268, 1970

3. Epstein NE: Advanced cervical spondylosis with ossification into the posterior longitudinal ligament and resultant neurologic sequelae. J Spinal Disord 9:477-484, 1996

4. Epstein NE: Ossification of the posterior longitudinal ligament: diagnosis and surgical management. Neurosurg Q 2:223-241, 1992

5. Epstein NE: Ossification of the posterior longitudinal ligament in evolution in 12 patients. Spine 19:673-681, 1994

6. Hanai K, Inouye Y, Kawai K, et al: Anterior decompression for myelopathy resulting from ossification of the posterior longitudinal ligament. J Bone Joint Surg Br 64:561-564, 1982

7. Harsh GH IV, Sypert GW, Weinstein PR, et al: Cervical spine stenosis secondary to ossification of the posterior longitudinal ligament. J Neurosurg 67:349-357, 1987

8. Hida K, Iwasaki Y, Koyanagi I, et al: [Surgical management of ossification of the posterior longitudinal ligament.] Spinal Surg 11:27-32, 1997 (Jpn)

9. Hirabayashi K, Miyakawa J, Satomi K, et al: Operative results and postoperative progression of ossification among patients with ossification of cervical posterior longitudinal ligament. Spine 6:354-364, 1981

10. Ichimoto H, Kawai S, Saika M: [The course of the extension of the ossified area in OPLL of the cervical spine.] Seikeigeka 44: 1132-1138, 1993 (Jpn)

11. Inoue $\mathrm{H}$, Ohmori $\mathrm{K}$, Ishida $\mathrm{Y}$, et al: Long-term follow-up review of suspension laminotomy for cervical compression myelopathy. J Neurosurg 85:817-823, 1996

12. Kawagishi T, Harada M: [Studies of the prevalence of the ossification of the posterior longitudinal ligament of the cervical spine in diabetic patients.] Clin Orthop Surg 14:718-722, 1979 (Jpn)

13. Kawaguchi H, Kurokawa T, Hoshino Y, et al: Immunohistochemical demonstration of bone morphogenetic protein-2 and transforming growth factor-beta in the ossification of the posterior longitudinal ligament of the cervical spine. Spine 17 (Suppl 3):S33-S36, 1992

14. Koga H, Hayashi K, Taketomi E, et al: Restriction fragment length polymorphism of genes of the $\alpha 2$ (XI) collagen, bone morphogenetic protein-2, alkaline phosphatase, and tumor necrosis factor- $\alpha$ among patients with ossification of posterior longitudinal ligament and controls from the Japanese population. Spine 21:469-473, 1996 
15. Kojima T, Waga S, Kubo Y, et al: Anterior cervical vertebrectomy and interbody fusion for multi-level spondylosis and ossification of the posterior longitudinal ligament. Neurosurgery 24:864-872, 1989

16. Kurata A, Tokiwa K, Kitahara I, et al: [Myelopathy caused by hypertrophy of the posterior longitudinal ligament (HPLL): case report.] No Shinkei Geka 15:651-655, 1987 (Jpn)

17. Manabe S, Nomura S: [Anterior decompression for ossification of the posterior longitudinal ligament of the cervical spine.] No Shinkei Geka 5:1253-1259, 1977 (Jpn)

18. Matsunaga S, Sakou T, Taketomi E, et al: Effects of strain distribution in the intervertebral discs on the progression of ossification of the posterior longitudinal ligaments. Spine 21: 184-189, 1996

19. Mizuno J, Nakagawa H: Outcome analysis of anterior decompressive surgery and fusion for cervical ossification of the posterior longitudinal ligament: report of 107 cases and review of the literature. Neurosurg Focus 10 (4):Article 6, 2001

20. Mizuno J, Nakagawa H, Hashizume Y: Analysis of hypertrophy of the posterior longitudinal ligament of the cervical spine, on the basis of clinical and experimental studies. Neurosurgery 49:1091-1098, 2001

21. Mizuno J, Nakagawa H, Isobe M: [Surgical results of anterior approach in ossification of the posterior longitudinal ligament in the cervical spine.] Spinal Surg 11:39-46, 1997 (Jpn)

22. Mizuno J, Nakagawa H, Iwata K, et al: Compression myelopathy due to hypertrophy of the posterior longitudinal ligament associated with herniated intervertebral discs. Neuroorthop 13:113-120, 1992

23. Motegi H, Yamazaki M, Goto S, et al: Proliferating cell nuclear antigen in hypertrophied spinal ligaments. Immunohistochemical localization of proliferating cell nuclear antigen in hypertrophied posterior longitudinal ligament of the cervical spine. Spine 23:305-310, 1998

24. Murakami N, Muroga T, Sobue I: Cervical myelopathy due to ossification of the posterior longitudinal ligamant: a clinicopathologic study. Arch Neurol 35:33-36, 1978

25. Nagashima C: Cervical myelopathy due to ossification of the posterior longitudinal ligament. J Neurosurg 37:653-660, 1972

26. Nakagawa H, Mizuno J: The pathophysiology and management of ossification of the posterior longitudinal ligament, in Barrow DL (ed): Perspectives in Neurological Surgery. St Louis: Quality Medical, 1992, Vol 3, pp 38-48
27. Nishi Y, Hirabayashi K, Satomi K: [Expansive open-door laminoplasty for cervical myelopathy caused by OPLL.] Seikei Geka 44:1219-1224, 1993 (Jpn)

28. Okazaki T, Takuwa Y, Yamamoto M, et al: Ossification of the paravertebral ligaments: a frequent complication of hypoparathyroidism. Metabolism 33:710-713, 1984

29. Sakou T, Taketomi E, Matsunaga S, et al: Genetic study of ossification of the posterior longitudinal ligament in the cervical spine with human leukocyte antigen halotype. Spine 16: 1249-1252, 1991

30. Takuwa Y, Matsumoto T, Kurokawa T, et al: Calcium metabolism in paravertebral ligamentous ossification. Acta Endocrinol 109:428-432, 1985

31. Trojan DA, Pouchot J, Pokrupa R, et al: Diagnosis and treatment of ossification of the posterior longitudinal ligament of the spine: report of eight cases and literature review. Am J Med 92:296-306, 1992

32. Watanabe I: [Clinical and radiological investigation of ossification in the cervical posterior longitudinal ligament.] Niigata Igakukai Zasshi 91:98-114, 1977 (Jpn)

33. Yamaura I: [A clinico-pathological study of ossifying process in cervical posterior longitudinal ligament.] Saigaiigaku 18: 651-662, 1975 (Jpn)

34. Yamaura I: [Intervertebral disc degeneration and posterior longitudinal ligament.] Spine Spinal Cord 3:205-212, 1990 (Jpn)

35. Yamazaki A, Homma T, Ishikawa S, et al: Magnetic resonance imaging and histologic study of hypertrophic cervical posterior longitudinal ligament. A case report. Spine 16:1262-1266, 1991

36. Yonezawa T, Tsuji H, Katoh Y, et al: [Progression of ossified area of the posterior longitudinal ligaments in the cervical spine after en bloc laminoplasty, in Investigation Committee Report on the Ossification of the Spinal Ligaments of the Japanese Ministry of Public Health and Welfare.] Tokyo: JMPHW, 1996, pp 299-302 (Jpn)

Manuscript received November 15, 2001

Accepted in final form January 2, 2002.

Address reprint requests to: Junichi Mizuno, M.D., Department of Neurological Surgery, Aichi Medical University, 21 Karimata Yazako Nagakute, Aichi-gun Aichi 480-1195, Japan. email: jmizuno@amugw.aichi-med-u.ac.jp. 